\title{
NIOBIUM SPUTTERED QUARTER WAVE RESONATORS
}

\author{
V. Palmieri, V.L. Ruzinov*, S.Yu. Stark*, \\ O.B. Malishev^, R. Preciso, L. Badan, A.M. Porcellato, J.S. Sokolowski ${ }^{\circ}$, \\ G.P. Buso, F. Poletto \\ INFN Laboratori Nazionali di Legnaro, I-35020 Legnaro (Padua), Italy
}

\begin{abstract}
The feasibility of Niobium sputtered Copper Quarter Wave Resonators has been proved. An extremely simple cathodic configuration for sputtering in QWR was constructed. High purity and uniformity superconducting Niobium thin films are obtainable in a single sputtering run. The sputtering process in a cavity has been investigated optimizing the superconducting properties of the deposit for the final RF tests of resonators.
\end{abstract}

\section{Introduction}

At Laboratori Nazionali di Legnaro, in the framework of $R$ \& $D$ of technologies going on in parallel to the construction of the ALPI Linac, a research activity aiming to prove the feasibility of Niobium Sputtered Copper Quarter Wave Resonators (Nb/Cu QWR) was initiated in 1988, encouraged by CERN results on electron cavities [1]. In order to investigate the Sputtering of high quality superconducting thin films of Niobium onto Oxygen Free High Conductivity Copper quarter wave cavities a laboratory fully devoted to this subject was crganized.

By obtaining high quality superconducting films with good thickness uniformity all inside the resonator we have shown beyond any doubt that the tricky shape of QWR's is absolutely not an obstacle to the application of the sputtering technology. A cryogenic facility for radiofrequency measurements was set up, so we were able to start the production of prototypes and the subsequent if test.

\section{The sputtering configuration}

The main criterion for the choice of the type of sputuering discharge and of the configuration design was the simplicity of the cathodic structure. In these optics, DC sputtering is certainly less tricky than if Sputtering and the Biased Diode Sputtering was preferred to the Magnetron sputtering. Indeed the several advantages of higher deposition rates and lower operating Ar pressures become negligible in comparison with the loss in thickness uniformity of the coating and with the technological complications in achieving a proper magnetic confinement of the discharge.

In order to get high thickness uniformity of the coating, several cathodic configurations were investigated $[2,3]$, all designed according to the principle that the cathode geometry should follow as faithfully as possible the shape of the cavity. Moreover we searched for a one-step process, because a thermal resistance can appear due to the the interface between two different layers.

The film thickness on the internal and external wall of the coaxial line and on the shorting plate were found unavoidably different, proving also that three different discharge regimes take place in these three regions. Nevertheless a thickness difference of only $15 \%$ was found with the configuration sketched in fig. 1, for a certain choice of sputtering parameters, in particular the Argon pressure.

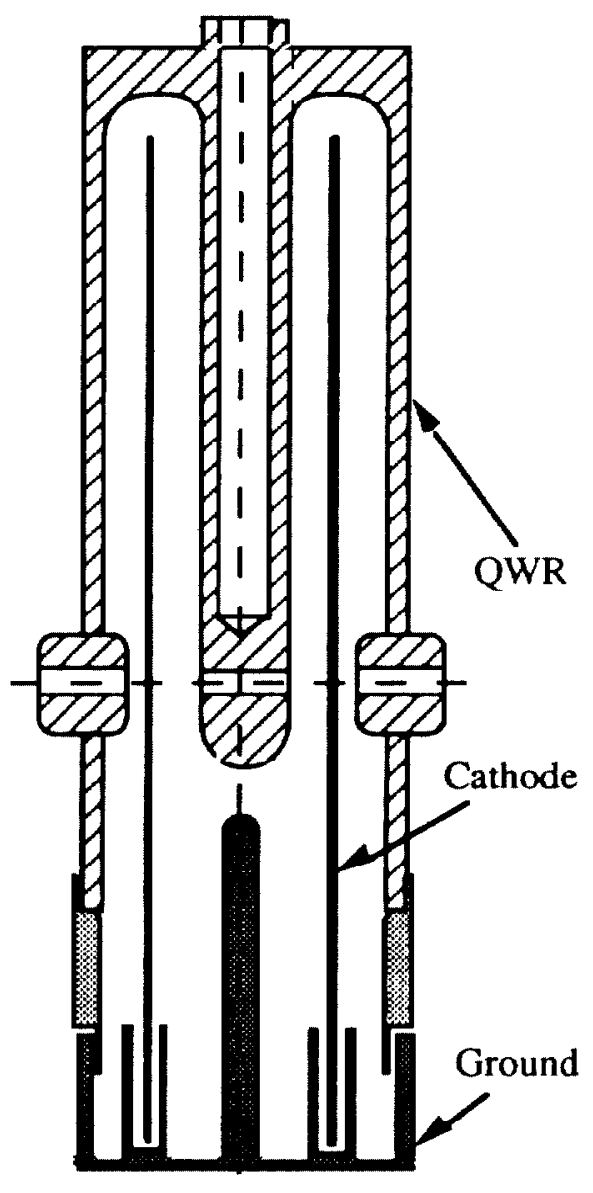

Fig.1 The sputtering configuration. The cavity is biased at negative potential.

Films of the order of $1 \mu \mathrm{m}$ thickness are generally deposited, since the smaller is the thickness, the less the

\footnotetext{
- On leave from Moscow Institute of Steel and Alloys, Moscow, Russia.

${ }^{\wedge}$ On leave from Institute of Nuclear Physics, Novosibirsk, Russia.

On leave from the Weizmann Institute of Science, Rehovot 76100, Israel.
} 
probability of film peeling and the higher the thermal stability of the $\mathrm{Nb} / \mathrm{Cu}$ system; a too small thickness however gives additional losses due to the penetration of RF fields into the Copper.

The problem of the beam ports coating has been already treated elsewhere $[2,3]$, and different variants were proposed. Choosing the most convenient among them will need further investigation.

\section{The sputtering process}

Once the cathoxie structure was established, around one hundred sputtering runs were performed onto a dummy cavity hosting Copper and sapphire samples in its interior, in order to find the right parameters at which to perform the process.

The surface quality of the Copper substrate after chemical polishing, the ultimate vacuum at which the sputtering chamber is evacuated before sputtering, the temperature of the resonator before and during the process, the Argon pressure, the discharge Voltage and Current, the bias Voltage of the substrate, the film thickness and the final rinsing of Niobium, all of them seriously influence the if performances of the resonator. It is interesting to note that two runs performed with exactly the same choice of these parameters show on samples rigourously the same distribution of the critical temperature Tc and of the Residual Resistivity Ratio RRR.

A standard choice for the sputtering parameters for the cylindrical configuration is the following:

$$
\begin{aligned}
& \text { Initial Pressure } \approx 10^{-8} \mathrm{mbar} \\
& \text { Argon pressure } \approx 2 \times 10^{-1} \mathrm{mbar} \\
& \text { Discharge Voltage } \approx-1.4 \mathrm{KV} \\
& \text { Discharge Current } \approx 5 \mathrm{~A} \\
& \text { Bias voltage }=100 \mathrm{~V} \\
& \text { Bias Current } \geq 3 \mathrm{~A} \\
& \text { Initial substrate tempcraturc } \approx 300^{\circ} \mathrm{C} \\
& \text { Final substrate temperature } \approx 800^{\circ} \mathrm{C}
\end{aligned}
$$

This set of numbers is not the only one possible. A small change around those does not give significant modifications to results. However we have observed that, since the sputtcring parameters are strongly correlated to each other, also other discharge regimes can lead to satisfactory results.

The sampling of the superconducting properties of the coating prove clearly that, among the plethora of parameters to control, three are the fundamental variables which more than others determine the high quality of the superconducting coating: the substrate temperature during the process, the rate of sputtering and the Bias voltage. In fact they significantly determine the Niobium film microstructure and purity since they directly affect the mechanism of grain growth and the sticking of impurities to the film during the process.

Fig. 2 shows the influence of the substrate temperature, the rate of deposition and the initial vacuum inside the sputtering chamber for the sputtering of the resonator bottom plate.

The distribution of RRR inside the resonator is displayed in fig. 3 .

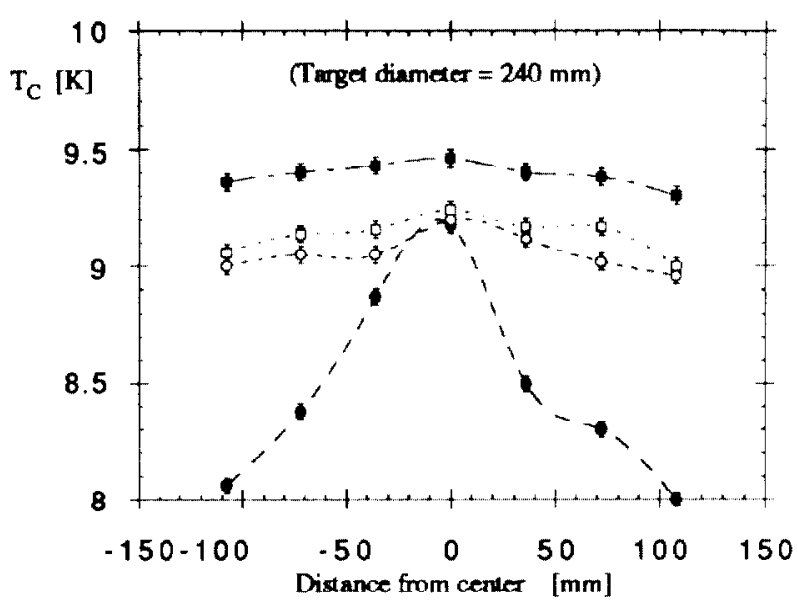

Fig. 2 The Tc distribution versus radius for the sputtered bottom plate of $180 \mathrm{~mm}$ diameter. The full circle curve refers to sputtering performed at a rate of $1.2 \mathrm{~A} / \mathrm{s}$. For the other three curves the rate was $7.8 \mathrm{~A} / \mathrm{s}$. The improvement from the empty circle curve to the empty square one is due to the lower residual pressure. The full squares curve refer to the case of an initial temperature of the Copper substrate of $300^{\circ} \mathrm{C}$, while for the previous curves refer to a substrate initially at room temperature

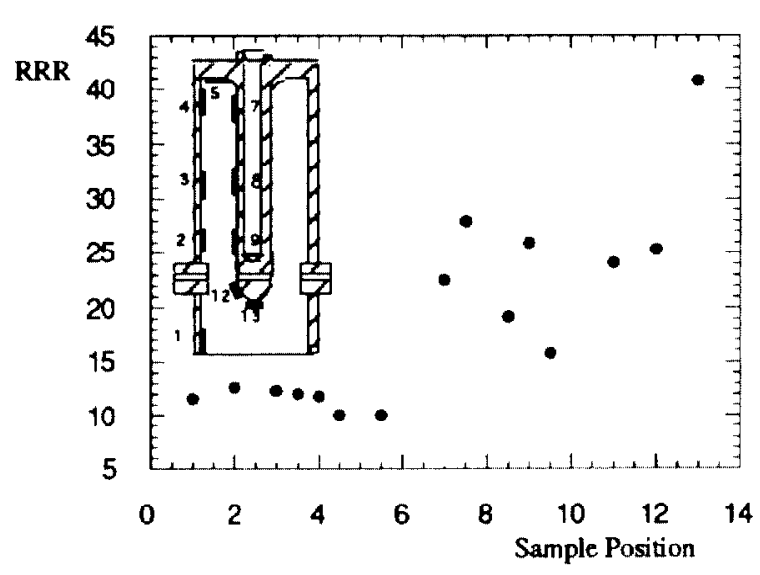

Fig. 3 The typical RRR distribution versus sample position inside the resonator.

The RRR values of samples of each run constitute a key for comparing various depositions. Our criterion for searching out the best sputtering parameters for the coating of Copper prototypes is that no place inside cavity should have $R R R<10$. Such a criterion is justified by the empirical correlation between $T c$ and RRR found for our films and shown in fig. 4. 


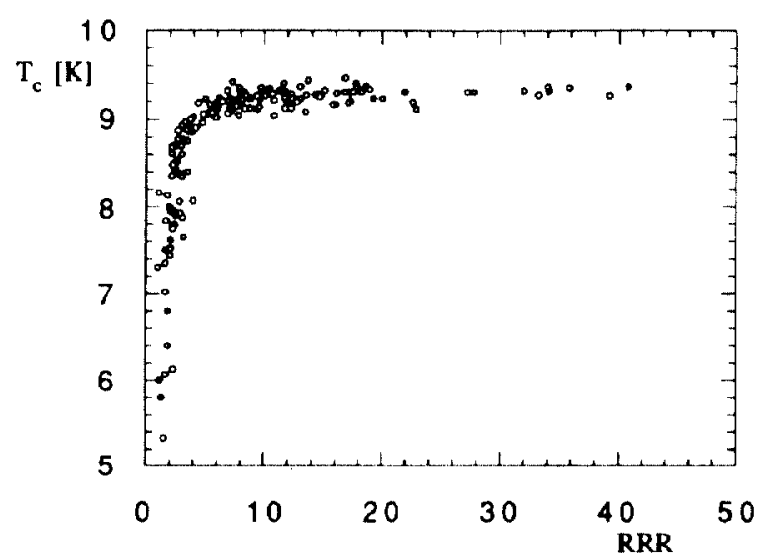

Fig. $4 T_{C}$ of Niobium sputtered on sapphire substrates versus the Residual Resistivity Ratio.

\section{The sputtering of the first Copper prototypes}

DC Biased Diode sputtering has been investigated in planar configuration for the sputtering of the bottom plate and in cylindrical configuration for the coaxial line. The problem of sputtering in cylindrical configuration of course presents more difficulties than the other one. So, at least in first approximation, we approached the problem, from the point of view of avoiding difficulties arising from the complex shape of cavity rather than fighting against them. For example we decided to study first a cavity without beam ports and moreover to move both the coupler and the pick up from the body of the cavity to the bottom plate. Hence a capacitive coupler was deviced and employed for RF measurements.

Up to now four prototypes were tested and the results are shown in fig.5.

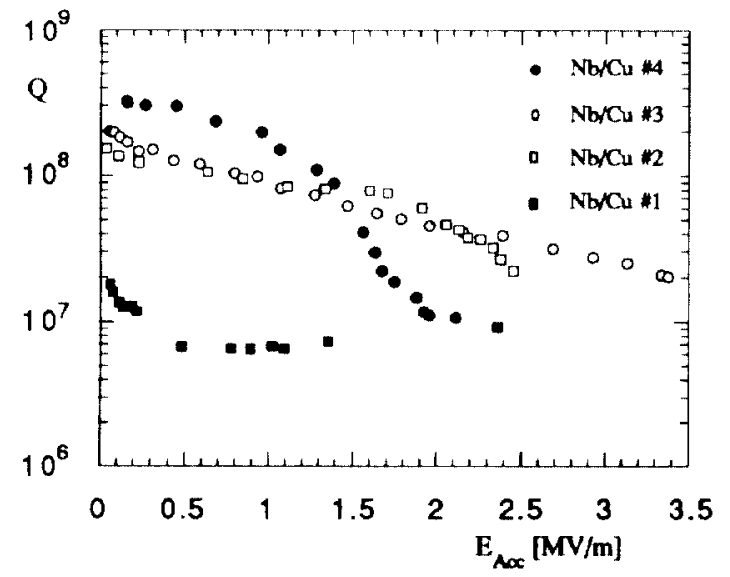

Fig. 5 The Q-factor versus accelerating field for the four $\mathrm{Nb} / \mathrm{Cu}$ prototypes produced.

A Q at zero field of $3.25 \cdot 10^{8}$ has been obtained for cavity \#4, while tan accelerating field of $2.8 \mathrm{MV} / \mathrm{m}$ at 7 Watt has been achieved by cavity \#3. We explain the constant decreasing of $Q$ when increasing the accelerating field of cavity $\# 3$ as due to a poor thermal conductivity of the interface between Niobium and Copper. On the other hand, we attribute the small value of the accelerating field of the cavity $\# 4$ to too small thickness of the Niobium coating.

The performances of such cavities did not yet achieve the design value for ALPI (at least $3 \mathrm{MV} / \mathrm{m}$ at $7 \mathrm{Watt}$, corresponding to a $Q$ around $8 \cdot 10^{7}$ ). Nevertheless the results are very encouraging. Indeed, test by test better values of $Q$ at zero field are obtained due to the constant improvement of our experience.

\section{References}

[1] C. Benvenuti, "Superconducting Coatings for accelerating RF cavities: past, present, future". Proceodings of the 5-th Workshop on RF Superconductivity, Hamburg, Germany, August 1991 .

[2] V. Palmieri, R. Preciso, V.L. Ruzinov, Ital. Pat. Appln. RM91 A000616 dep. on 14th August 1991.

[3] V. Palmieri, R. Preciso, V.L. Ruzinov, S.Yu. Stark, L. Badan, A.M. Porcellato, "Sputtering of Niobium thin films onto Copper Quarter Wave Resonators", ibid. ref. [1]. 